\title{
Dickkopf-3 (Dkk3) induces apoptosis in cisplatin-resistant lung adenocarcinoma cells via the Wnt/ß-catenin pathway
}

\author{
ZHENG WANG $^{1 *}$, LI-JUN MA $^{1 *}$, YI KANG $^{2}$, XIAO LI ${ }^{1}$ and XIAO-JU ZHANG ${ }^{1}$ \\ ${ }^{1}$ Department of Respiratory Medicine, The People's Hospital of Zhengzhou University, Zhengzhou, Henan 450003; \\ ${ }^{2}$ Department of Infectious Disease, The People's Hospital of Zhengzhou University, Zhengzhou, Henan 450000, P.R. China
}

Received May 29, 2014; Accepted September 11, 2014

DOI: 10.3892/or.2014.3704

\begin{abstract}
Previous studies have shown that Dickkopf-3 (Dkk3) is inactivated in lung cancer cells, while the inactivation of the Wnt/ $\beta$-catenin signaling pathway by $\mathrm{Dkk} 3$ inhibits lung cancer progression. In the present study, we investigated whether Dkk3 enhances the sensitivity of lung cancer cells to cisplatin. A549, Calu1 and H460 lung adenocarcinoma cell lines were transfected with DKK3 siRNA, while the cisplatin-resistant subline A549cis was transfected with DKK3. DKK3 expression was attenuated in A549cis, Calu1cis and H460cis compared to A549, Calu1 and H460, respectively. Lung adenocarcinoma cell growth, proliferation, apoptosis, cell cycle in vitro and in vivo were then analyzed. DKK3 knockdown by siRNA transfection rendered A549, Calu1 and H460 resistant to cisplatin. As a result of DKK3 transfection, the expression of DKK3 and E-cadherin was significantly upregulated, while that of MMP7, survivin, c-myc and cyclin D1 was downregulated. DKK3 overexpression retarded cell proliferation, induced cell cycle arrest and apoptosis, and reduced cell invasive ability in the A549 and A549cis cells. In addition, the proportions of apoptotic cells and the PARP level were significantly increased in A549cis- and H460cis-DKK3 cells treated with cisplatin. Moreover, tumor growth was retarded more in cisplatin-treated nude mice seeded with A549cis-DKK3 cells than with A549cis cells. Cell viability increased with the pretreatment of SB216763 for $2 \mathrm{~h}$ in A549cis and A549cis-DKK3 cells incubated with cisplatin $(1 \mu \mathrm{M})$
\end{abstract}

Correspondence to: Professor Xiao-Ju Zhang, Department of Respiratory and Critical Care Medicine, Henan Provincial People's Hospital, No. 7 Weiwu Road, Zhengzhou, Henan 450003, P.R. China E-mail: zhangxiaoju1010@hotmail.com

${ }^{*}$ Contributed equally

Abbreviations: DKK/Dkk, Dickkopf; NSCLC, non-small cell lung cancer; PARP, poly(ADP-ribose) polymerase; REIC, reduced expression in immortalized cells

Key words: lung cancer, cisplatin, resistance, Dickkopf3, survivin for $72 \mathrm{~h}$. In conclusion, the re-activation of Dkk3 enhances the chemosensitivity to cisplatin in cisplatin-resistant lung adenocarcinoma cell lines, which requires additional studies to realize this potential in clinical use.

\section{Introduction}

Lung cancer has emerged as the third leading cause of cancer-related mortality worldwide (1). In the USA, over 200,000 new lung cancer cases are diagnosed annually, causing over 150,000 deaths in one year (1). Approximately $85 \%$ of lung cancer patients suffer from non-small cell lung cancer (NSCLC). Due to the comparative therapeutic advantage, cisplatin-based combination regimens are recommended as the optimal choice currently for the majority of NSCLC patients indicative of chemotherapy or adjuvant chemotherapy (2). However, the average survival time for patients with advanced stage of NSCLC receiving cisplatin plus gemcitabine treatment is 16 months and may even be reduced to 12 months in those with cisplatin-resistance (3). The dilemma in managing late-stage NSCLC requires the elucidation of the mechanisms in cisplatin resistance to define novel, effective and applicable therapeutic targets for lung cancer $(2,3)$.

Several signal transduction pathways controlling chemosensitivity are aberrantly activated in various types of cancer, among which Wnt is of special significance (4). The role of Wnt1/Int-1 in the induction of mouse mammary tumor as an integration site for mouse mammary tumor virus (MMTV) was first recognized 30 years ago (4). Wnt/ $\beta$-catenin signaling is initiated by the binding of secreted Wnt proteins with the frizzled, a class of seven-pass transmembrane receptors. Activation of the receptor leads to the phosphorylation of the dishevelled protein which, through its association with axin, prevents glycogen synthase kinase $3 \beta$ (GSK3 $\beta$ ) from phosphorylating $\beta$-catenin and the negative regulators axin and adenomatous polyposis coli (APC). Unphosphorylated $\beta$-catenin escapes recognition, ubiquitinization and degradation by $\beta$-TRCP, accumulates in the cytoplasm and translocates to the nucleus, where it engages with transcription factors such as TCF and LEF. The nuclear accumulation of $\beta$-catenin switches on the TCF/LEF-controlled transcription of downstream genes. Wnt effector genes include E-cadherin, MMP7, survivin, c-myc and cyclin D1, all of which control the fate of cancer development, progression and metastasis $(4,5)$. 
Abnormal activation of the $\mathrm{Wnt} / \beta$-catenin signaling pathway has been most extensively studied in colorectal neoplasias, and was realized further in almost all types of solid organ and haematologic malignancies in human beings, including lung adenocarcinoma (6). Wnt inhibition, either by monoclonal antibodies, small-interfering RNAs (siRNAs) targeting Wnt components or the overexpression of Wnt antagonists, retards lung cancer progression in various in vitro and in vivo tumor models $(5,6)$. Furthermore, inactivation of $\mathrm{Wnt} / \beta$-catenin signaling sensitizes chemotherapy by inducing apoptosis and growth arrest in cancer cells, suggesting the potential role of Wnt signaling inhibitors in the reversal of cisplatin resistance (7-9).

As a soluble Wnt inhibitor, Dickkopf-3 (Dkk3) is involved in molecular cancer therapy. Dkk3 binds with LDL-receptor-related protein5/6 (LRP5/6) and destabilizes cytoplasma $\beta$-catenin (10). Dkk3 is downregulated in a variety of malignancies including hepatic cancer, kidney carcinoma, urinary bladder cancer, pancreatic cancer and lung cancer, earning its alias, the 'reduced expression in immortalized cells' (REIC) (11). Re-established Dkk3/REIC expression induces apoptosis in cancer cell lines (12-14). Downregulation of Dkk3/REIC through epigenetic hypermethylation is universal among lung cancer cell lines and human lung cancer samples $(15,16)$. While DKK3 knockdown stimulates the proliferation of lung cancer cells, DKK3 overexpression inhibits the growth of NSCLC cells by inducing apoptosis and cell cycle disturbance via the transactivation of c-myc and cyclin D1 through $\beta$-catenin/TCF-4 signaling (17). The abovementioned emphasize the tumor suppressive role of $\mathrm{Dkk} 3$ with capacities of promoting apoptosis and inhibiting proliferation in lung cancer cells (18). The possible chemosensitizing effect of Dkk3 is suggested as its homologue, Dkk1, has shown a pro-apoptotic activity that inhibits the growth synergistically with cisplatin in cisplatin-resistant head-neck and brain tumor cells $(19,20)$.

The present study aimed to test the hypothesis that Dkk3 may disturb the growth of lung cancer cells synergistically with cisplatin. DKK3 expression levels in wild-type and cisplatin-resistant NSCLC cell lines were subsequently transfected with DKK3 siRNA or DKK3 gene. We then investigated whether the downregulation or upregulation of DKK3 was able to disrupt the growth of cisplatin-resistant NSCLC cells treated with cisplatin in vitro and in vivo. To determine the possible chemosensitization mechanisms of Dkk3 functioning, a small-molecular Wnt activator was allocated prior to cisplatin treatment (21). Then we compared the biological behavior of cisplatin-resistant NSCLC cells with or without DKK3 transfection, and the expression profile of genes and proteins downstream of the Wnt signaling pathway, which possibly regulate the cell cycle, cell proliferation and apoptosis. The results may provide substantial evidence in support of the therapeutic value of DKK3 for lung cancer refractory to chemotherapy.

\section{Materials and methods}

Cell culture. Immortalized HEK293 and A549, Calu1 and H460 human lung adenocarcinoma cell lines were purchased from the American Type Culture Collection (ATCC; Manassas,
VA, USA). These cell lines were tested and authenticated prior to using short tandem repeat (STR) loci by the Cell ID System (Progema, Madison, WI, USA). Cisplatin-resistant cell sublines, A549cis, Calu1cis and H460cis, were generated by continuous exposure to increasing concentrations of cisplatin (from 10 to $50 \mu \mathrm{M}$ ) for 12 months as previously described (21). The cells were incubated at $37^{\circ} \mathrm{C}$ with $5 \% \mathrm{CO}_{2}$ in atmosphere and grown in RPMI-1640 supplemented with $10 \%$ fetal bovine serum, $1 \%$ penicillin/streptomycin, and 5\% MEM non-essential aminoacids (HyClone Laboratories, Waltham, MA, USA). The medium was restored three times a week, and subcultures were performed prior to cells reaching 60-70\% confluence. To identify the impact of GSK3 $\beta$ inactivation on the concentration-effect curves with cisplatin, A549cis and A549cis-DKK3 cells were treated with a GSK3 $\beta$ inhibitor SB216763 [3-(2, 4-dichlorophenyl)-4-(1-methyl-1H-indol-3-y 1)-1H-pyrrole-2,5-dione] $2 \mathrm{~h}$ prior to cisplatin exposure (22).

DKK3 RNA interference. siRNA of DKK3 and a control scrambled RNA targeting a sequence not sharing homology with the human genome (negative control) was synthesized (Shanghai Invitrogen Ltd., Shanghai, China). The oligonucleotide sequences targeting DKK-3 were: 5'-AAUGGUCUGGU ACUUAUUCCdGdC-3' (forward), and 3'-dCdGUUACCAG ACCAUGAAUAAGG-5' (reverse). Calu1 and H460 lung carcinoma cells were incubated with siRNAs and control scrambled RNA using the Lipofectamine RNAiMAX reagent (Shanghai Invitrogen Ltd.). The procedures were performed according to the manufacturer's instructions. After transfection for $6 \mathrm{~h}$, the transfection mixture was removed and the cells were incubated for 1-3 x $24 \mathrm{~h}$ prior to detection.

Plasmid construction and DKK3 transfection. Genomic DNA was isolated from A549 lung cancer cell lines using the All Prep DNA/RNA Mini Kit (Qiagen). The bisulfite modification of genomic DNA was carried out with the EpiTect Bisulfite kit (Qiagen) according to the manufacturer's instructions. Primers for bisulfite genomic sequencing PCR were designed manually or by using the online MethPrimer program. The DKK3 primers used were: forward, 5'-GGAGGAGGTTATTTTT AATGAGATGT-3' and reverse, 5'-TCCAAACTTTTTAACA AAAAAACAAA-3'. The amplification products were sequenced directly by an outside vendor (McLab, San Francisco, CA, USA). The full-length cDNA was integrated into a cosmid vector $\mathrm{pAxCAwt}$ and transferred into an adenovirus vector by the COS-TPC method (Takara Bio, Shiga, Japan). An adenovirus vector carrying the LacZ gene was used to monitor infection efficiency. A549 and cisplatin-resistant A549cis lung carcinoma cells were incubated with DMSO, Ad-DKK3 or Ad-GFP at various multiplicity of infections (MOIs) (ranging from 0, 1, 5, 10, 20, 50 to 100). The procedures were performed according to the manufacturer's instructions. After incubation for $6 \mathrm{~h}$, the transfection mixtures were removed. Clonal cells were nominated as A549-AdV, A549-DKK3, A549cis-AdV and A549cis-DKK3. The cells were incubated for another 1-6 x $24 \mathrm{~h}$ prior to detection or in vivo injection.

Reverse transcriptase-polymerase chain reaction (RT-PCR). Total RNA was isolated using TRIzol reagent (Invitrogen) 
according to the manufacturer's instructions. The primer sequences used were: DKK3, 5'-ATGCAGCGGCTTGGG GCCACCCTGCTGTGC-3' (forward), and 5'-GATGGTCCCA TTGCTGCCCCTGGTGGCCAT-3' (reverse); Wnt1, 5'-TGGT TTGCAAAGACCACCTCCA-3' (forward), and 5'-TGATTCC AGGAGGCAAACGCAT-3' (reverse); Wnt3a, 5'-TGGTTTGC AAAGACCACCTCCA-3' (forward), and 5'-TGATTCCAGG AGGCAAACGCAT-3' (reverse); c-myc, 5'-CTGCGACGAG GAGGAGGACT-3' (forward), and 5'-GGCAGCAGCTCG AATTTCTT-3' (reverse); E-cadherin, 5'-TCCCATCAGC TGCCCAGAAA-3' (forward), and 5'-TGACTCCTGTGT TCCTGTTA-3' (reverse); and MMP-7, 5'-GGTCACCTACAG GATCGTATCATAT-3' (forward), and 5'-CATCACTGCAT TAGATCAGAGGAA-3' (reverse). cDNA synthesis was normalized by PCR with GAPDH primers: 5'-CATCACTGC CACCCAGAAGA-3' (forward), and 5'-TGAAGTCGCAGGA GACAACC-3' (reverse). PCR conditions were as follows: 45 cycles of $30 \mathrm{sec}$ at $95^{\circ} \mathrm{C}, 30 \mathrm{sec}$ at $58^{\circ} \mathrm{C}$, and $60 \mathrm{sec}$ at $72^{\circ} \mathrm{C}$. Specificity of amplification products was verified by agarose gel electrophoresis.

Western blot analysis. Antibodies against $\beta$-catenin, Fas, caspase-3, cleaved caspase-3, caspase-8, cleaved caspase-8, survivin, $\beta$-actin and poly(ADP-ribose) polymerase (PARP) were produced by Santa Cruz Biotechnology, Inc. (Santa Cruz, CA, USA). Protein lysate was separated electrophoretically on denaturing SDS-polyacrylamide gel, transferred to nitrocellulose membranes, and probed with goat polyclonal IgG antibodies (1:200 or 1:250 diluted). Blots were exposed to secondary antibodies and detection was performed using an enhanced chemiluminescence reagent (Amersham). For the loading control, the membrane used in the initial western blotting was placed in Restore Western blotting Stripping Buffer (Thermo Scientific) for $15 \mathrm{~min}$ to remove the antibody (primary and secondary antibodies). The membrane was then washed with water for $5 \mathrm{~min}$, blocked with $5 \%$ milk for $1 \mathrm{~h}$, and probed with $\beta$-actin.

Cell proliferation assay. Cell viability/proliferation assays were performed using the Cell Counting Kit-8 assay (CCK-8; Dojindo Molecular Technologies, Shanghai, China) which uses the bioreduction of WST- 8 to orange-colored formazan to measure cell viability. Briefly, $100 \mu 1$ of cells at $2 \times 10^{4} /$ $\mathrm{ml}$ cells were plated on 96-well culture plates. NSCLC cells transfected with DKK3siRNA or DKK3 were cultured in cisplatin (Sigma-Aldrich, St. Louis, Mo, USA). At various time-points over the next $72 \mathrm{~h}$, the culture medium in quadruplicate wells was replaced with fresh medium containing 5\% CCK- 8 reagent, and after $1 \mathrm{~h}$, absorbance at $450 \mathrm{~nm}$ was measured on a Multiskan Plus (MTX Lab Systems ${ }^{\circledR}$, Vienna, VA, USA) plate reader. Absorbance values were corrected by subtracting those of blank control wells that did not have cells. The linear regression equations that correlated log (cisplatin concentration) with cell viability were established by linear regression. The $\mathrm{IC}_{50}$ values (50\% inhibitory concentration) of cisplatin for each condition were then calculated by applying the logarithmic equations. The initial cisplatin concentration gradient was $0,0.05,0.1,0.2,0.4,0.8,1.6,3.2$, $6.4,12.8,25.6$ and $51.2 \mu \mathrm{M}$, and was subsequently adjusted based on the $\mathrm{IC}_{50}$ value.
Transwell invasion assay. Cell invasion assays were performed using 24-well Transwell (Corning Life Sciences) coated with Matrigel (1 mg/ml, BD Sciences). Cells (10\%/well) were seeded in the upper chambers of the wells in $200 \mu 1$ FBS-free medium, and the lower chambers were filled with $500 \mu 110 \%$ FBS medium to induce cell migration. Following incubation for $24 \mathrm{~h}$, the cells on the filter surface were fixed with $4 \%$ formaldehyde, stained with $0.5 \%$ crystal violet, and examined under a microscope. Cells in at least five random microscopic fields (x200) were counted.

Immunofluorescence. The expression of Dkk3 or $\beta$-catenin in NSCLC cells was investigated by immunofluorecent staining. Cells were plated onto a cover glass $(1 \times 1 \mathrm{~cm})$ placed in a 6-well culture plate at a density of $1 \times 10^{5}$ cells/well. Cover glasses were washed with $0.1 \mathrm{M}$ phosphate-buffered saline (PBS), fixed in ice-cold acetone for $5 \mathrm{~min}$, and soaked in PBS containing $0.2 \%$ Triton $\mathrm{X}-100$ for $5 \mathrm{~min}$ to increase their permeability to primary antibodies. The glasses were rinsed in PBS for $15 \mathrm{~min}$ and blocked with $5 \%$ bovine serum albumin at room temperature for $30 \mathrm{~min}$, then conjugated with primary antibodies (1:200) overnight at $4^{\circ} \mathrm{C}$. After washing three times with PBS, the cover glasses were incubated with fluorescein isothiocyanate (FITC)-conjugated secondary antibodies (1:40) at room temperature for 4-6 $\mathrm{min}$. The glasses were then double-stained with $0.5 \mu \mathrm{g} / \mathrm{ml} \mathrm{4',6-diamidino-2-phenylindole} \mathrm{(DAPI)} \mathrm{for}$ 5 min, and sealed with glycerol. Immunofluorescent images were captured using a Nikon C1si multifocal one-photon laser scanning microscope (Nikon Ltd., Tokyo, Japan) within $30 \mathrm{~min}$. Primary and secondary antibodies were purchased from Santa Cruz Biotechnology, Inc. DAPI was from Sigma-Aldrich Co.

Flow cytometric analysis. The cells were plated at a density of $1 \times 10^{5}$ cells/well in 6 -well plates. The cell cycle distribution of cells stained with propidium iodide (PI) was analyzed. Apoptotic events were measured by Annexin V-FITC double staining according to the manufacturer's instructions (Nexins Research, Kattendijk, The Netherlands). Analyses were performed on a FACSCalibur instrument using the CellQuest or the ModFit 3.0 software packages (Becton-Dickinson, Mount View, CA, USA). Apoptosis was also manifested by detecting PARP cleavage by immunoblot as mentioned above.

In vivo tumorigenesis. Experimental procedures were approved by the Animal Care and Use Committee of Zhengzhou University, and were in accordance with the Guide for the Care and Use of Laboratory Animals issued by the USA National Institutes of Health. Four-week-old male $\mathrm{nu} / \mathrm{nu}$ nude mice, weighing $19.3 \pm 2.2 \mathrm{~g}$, obtained from the Shanghai Institute of Drug of Chinese Academy of Sciences, were housed in specific pathogen-free conditions. A549cis, A549cis-AdV or A549cis-DKK3 cells were grown to near confluence, digested and resuspended in PBS. PBS $(0.1 \mathrm{ml})$ containing $1 \times 10^{6}$ cells was then injected subcutaneously into the flanks of nude mice. Cisplatin was administered via intraperitoneal injection at doses of $2.5 \mathrm{mg} / \mathrm{kg} / \mathrm{day}$ for 5 days/week. The tumor size was measured using a caliper. The tumor volume was calculated according to the formula: $\mathrm{V}=1 / 6 \pi \mathrm{ab}^{2}(\pi, 3.14$; $\mathrm{a}$, long axis; and $\mathrm{b}$, short axis of the tumor). Growth curves were plotted from the mean tumor 
volume \pm SD from 10 animals in each group. Six weeks after the injection, the animals were sacrificed and tumors were harvested, measured, weighed and fixed in $10 \%$ formalin. The wet tumor weight of each animal was calculated as means \pm SD from 10 animals in each group.

Statistical analysis. Data were analyzed by the SPSS 15.0 software (SPSS, Inc., Chicago, IL, USA) and SigmaPlot 12.0 (Systat Software Ltd., Chicago, IL, USA). The Student's t-test and one-way ANOVA were used to examine the differences between or among groups. The Kruskal-Wallis rank sum test was used alternatively when data were inappropriate for ANOVA analysis (e.g., without homogeneity of variance). Statistical tests were two-sided. $\mathrm{P}<0.05$ was considered to indicate a statistically significant result.

\section{Results}

Decreased expression of DKK3 is associated with cisplatin resistance in lung cancer cells. The cisplatin-resistant A549cis, Calulcis and H460cis NSCLC sublines, were characterized by the WST- 8 viability assay. Dkk3 expression was markedly reduced in Calulcis (0.23-fold compared with Calu1) and less in H460cis (0.41-fold compared with Calu1) (Fig. 1). A549 expressed low levels of Dkk3, with RT-PCR analysis shown a 1.2-fold decrease in DKK3 mRNA with A549cis (data not shown). The impact of DKK3 knockdown (by siRNA transfection) on cisplatin-induced growth arrest was determined. Compared to scrambled RNA transfection, DKK3 knockdown ameliorated cisplatin-induced growth arrest in the three NSCLC cell lines, and rendered them resistant to cisplatin (Fig. 2A and B). As shown in Fig. 2C and D, the viability and proliferation index of Calu1-DKK3ko and H460-DKK3ko
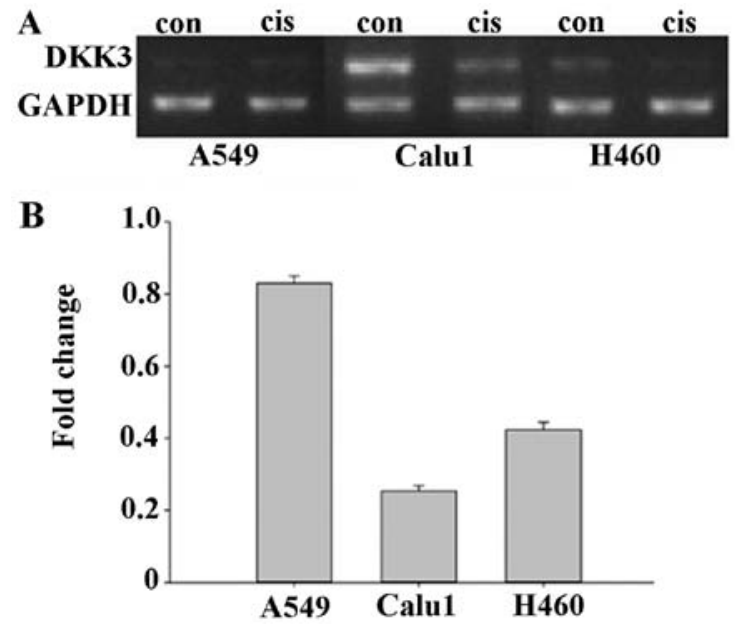

Figure 1. Decreased expression of DKK3 is associated with cisplatin resistance in lung cancer cells. (A and B) DKK3 expression was inhibited in cisplatin-resistant NSCLC cell lines as compared with their naive counterparts. DKK3, Dickkopf-3; NSCLC, non-small cell lung cancer.

were increased as compared with Calu1 and $\mathrm{H} 460$ at the same cisplatin concentrations $(\mathrm{P}<0.05$ for both).

DKK3 transfection inhibits NSCLC cell growth. As shown in Fig. 3, the DKK3 transgene altered the cell cycle in A549 cells, and attenuated A549 cell mobility. An increase in the portion of cells in the G0/G1 phase of the cell cycle by $17-20 \%$ in A549cis cells transfected with DKK3 gene and with a corresponding decrease in cells in the S and G2-M phase was observed. By contrast, for A549cis transfected with the empty vector, no significant changes in cell cycle distribution were detected (Fig. 3). Cell invasion assay was performed
A
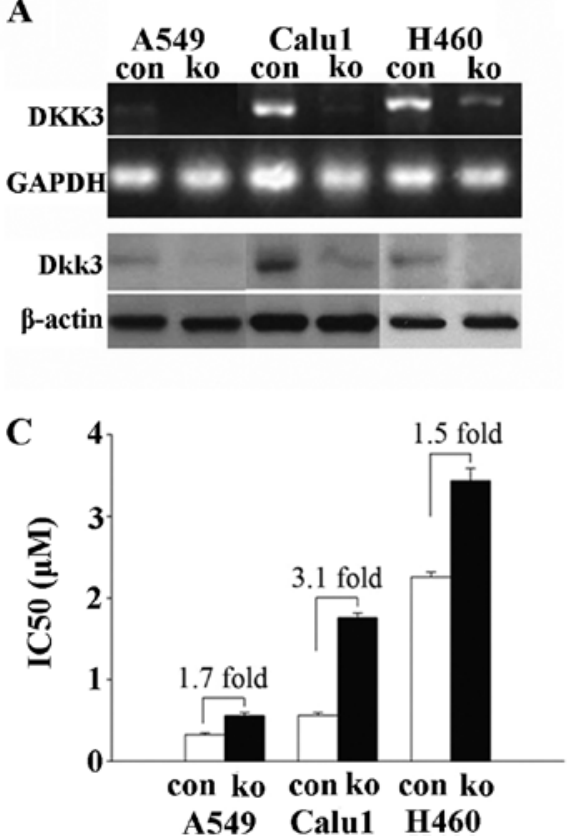
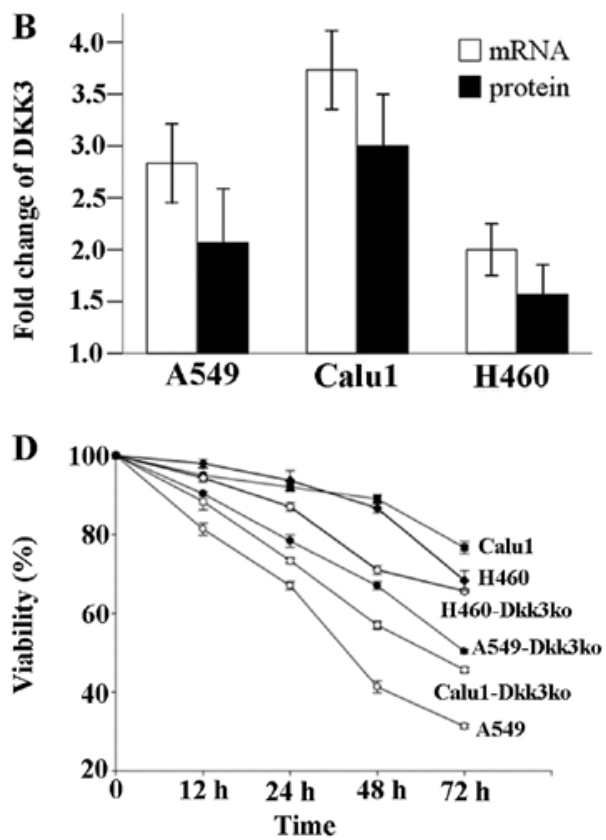

Figure 2. DKK3 knockdown mediates cisplatin resistance. (A and B) The impact of DKK3 knockdown (by siRNA transfection) on cisplatin-induced growth arrest. (C) $\mathrm{IC}_{50}$ fold changes after DKK3 knockdown in NSCLC cells. (D) Time-viability curve in the three NSCLC cell lines and their Dkk3-knockout (Dkk3ko) sublines exposed to cisplatin (1 $\mu \mathrm{M}$ for each time). Data (means $\pm \mathrm{SD}$ ) are representative of three independent experiments. DKK3, Dickkopf-3; NSCLC, non-small cell lung cancer. 

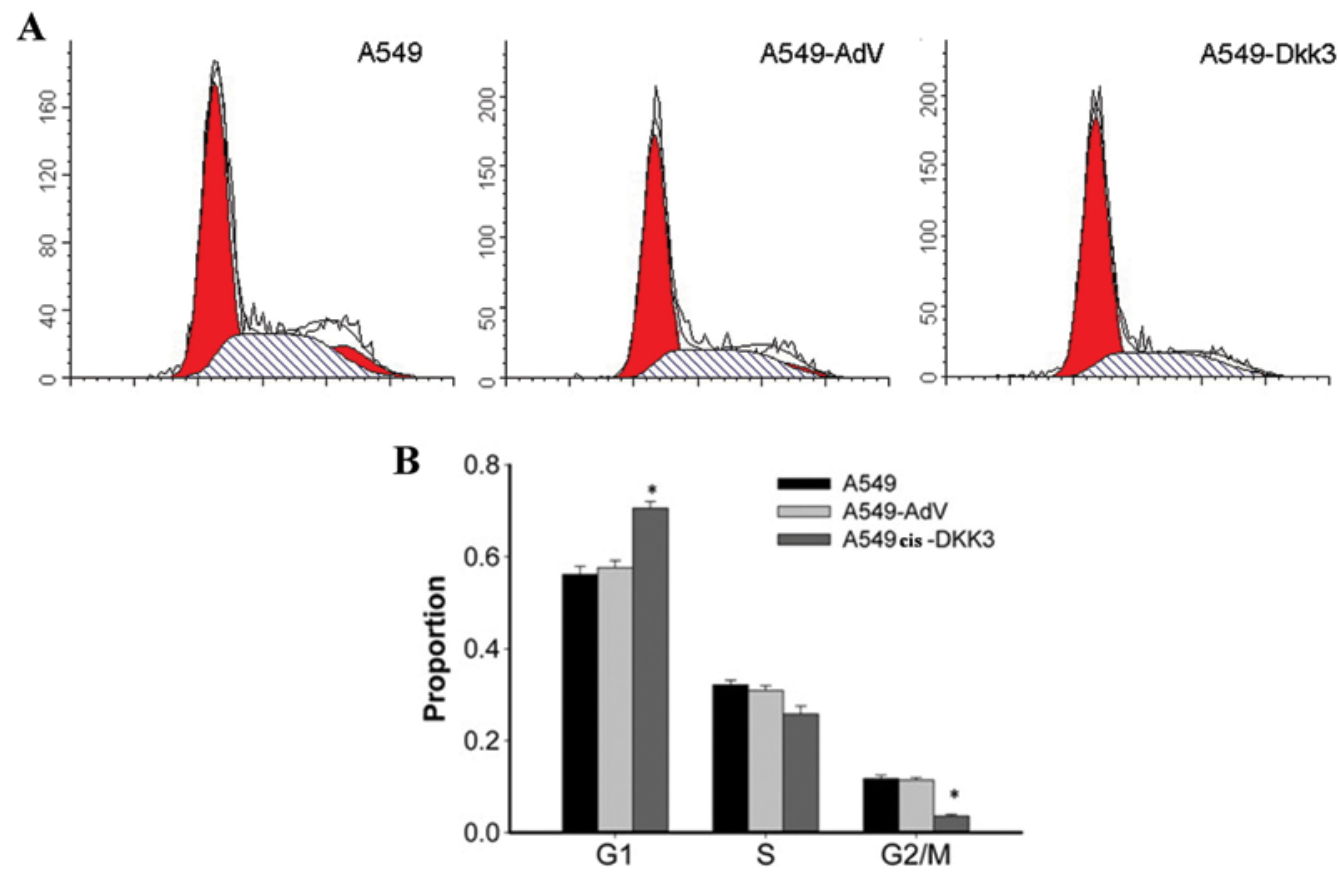

Figure 3. DKK3 transfection induced cell cycle derangement in NSCLC cells. (A) Cell cycle status in A549, A549-AdV and A549-Dkk3. (B) Statistical analysis of cell cycle proportions in NSCLC cells. DKK3, Dickkopf-3; NSCLC, non-small cell lung cancer.
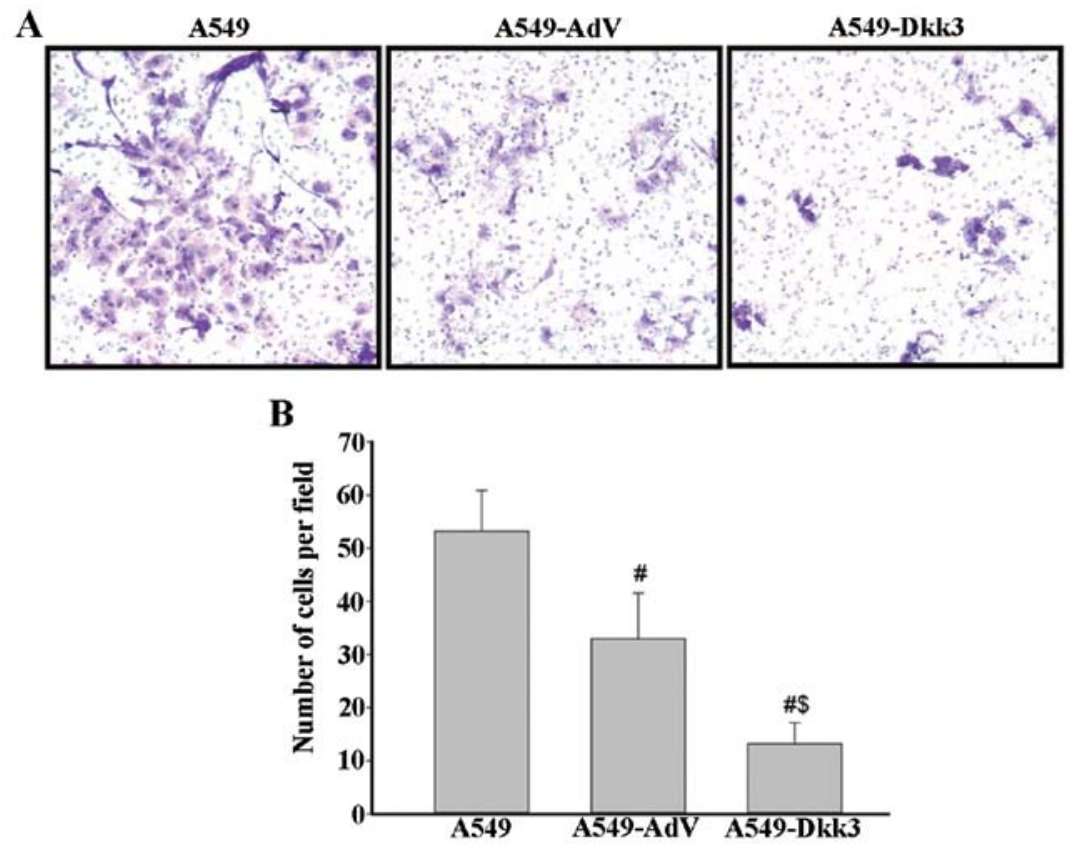

Figure 4. DKK3 transfection reduced the mobility of lung cancer cells. (A) The mobility of lung cancer cells was analyzed on Matrigel. (B) Statistical analysis of penetrating NSCLC cells per high magnified field. Data are representative of three independent experiments. ${ }^{\#} \mathrm{P}<0.05$. DKK3, Dickkopf-3; NSCLC, non-small cell lung cancer.

using a 24-well Transwell. Ectopic expression of DKK3 significantly reduced the number of cells migrating through the Matrigel-coated filters (Fig. 4). Cell apoptosis was assayed by flow cytometry with Annexin V-FITC staining. Overexpression of DKK3-induced apoptosis of lung cancer cells as compared to scramble sequence-transfected cells in A549 cell lines (Fig. 5A and C). The cleaved PARP level was also significantly elevated with DKK3 transfection
(Fig. 5B and D). These data suggest that DKK3 alone inhibits NSCLC cell growth.

DKK3 transfection plus cisplatin synergistically suppresses the growth of resistant NSCLC cells in vitro and in vivo. We transfected A549cis with DKK3, and assessed the efficiency of DKK 3 transfection by RT-PCR and immunofluorescence. As a consequence of DKK3 transfection, DKK3 was localized 


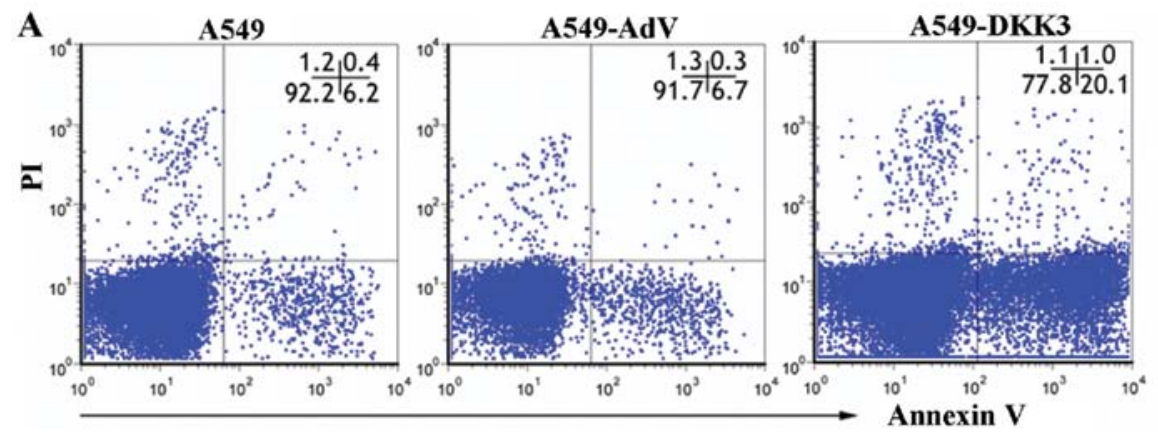

B $\quad$ A549 A549-DKK3 A549-AdV
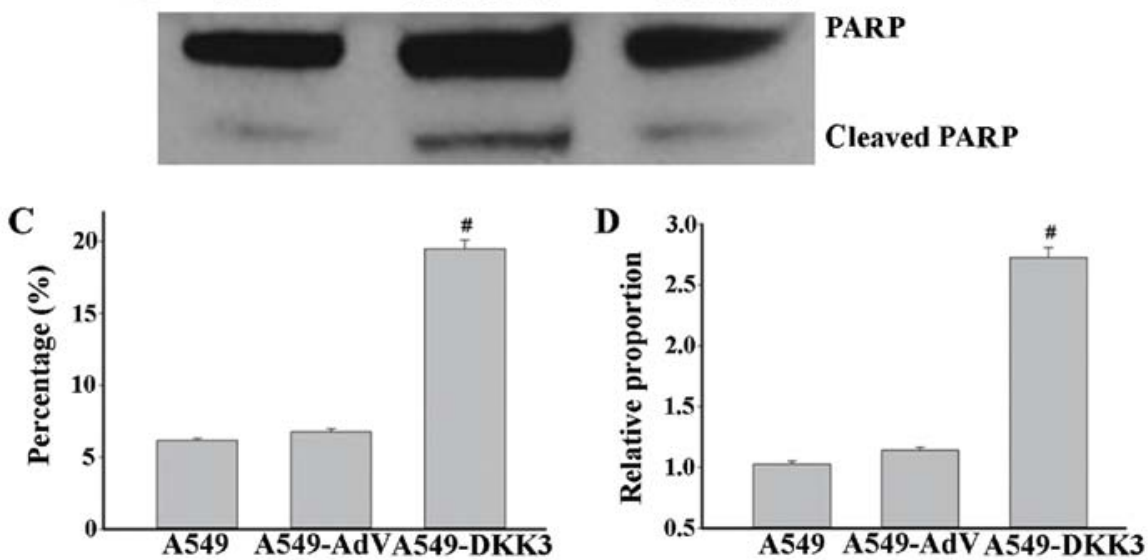

Figure 5. DKK3 transfection enhanced apoptosis in A549 cells. (A and C) Cell apoptosis assayed by flow cytometry with Annexin V-FITC staining. (B and D) Cleaved PARP level was also significantly elevated with DKK3 transfection. Data are representative of three independent experiments. "P<0.05. DKK3, Dickkopf-3.
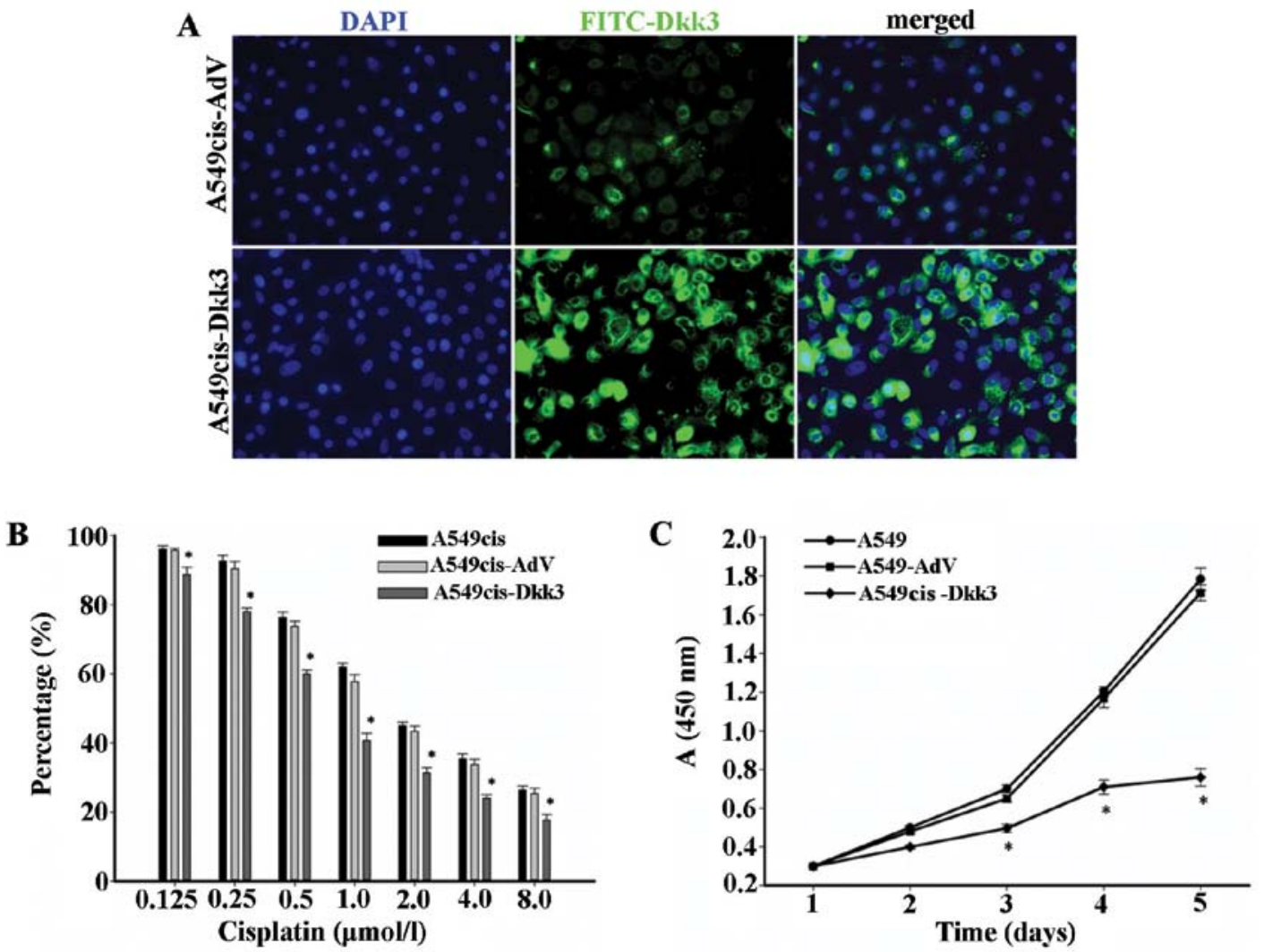

Figure 6. DKK3 transfection inhibits cisplatin-resistant lung cancer cell growth in vitro. (A) DKK3 immunofluorescence in vitro. DKK3 was localized mostly at the cytoplasm, following staining with FITC-conjugated secondary antibody (green). The nuclei appear as blue fluorescence stained with DAPI (original magnification, x100). (B) A549cis, A549-AdV and A549-DKK3 cells treated with cisplatin for $72 \mathrm{~h}$. (C) Time-viability curve of NSCLC cells treated with cisplatin $(1 \mu \mathrm{M})$. DKK3, Dickkopf-3; NSCLC, non-small cell lung cancer. 

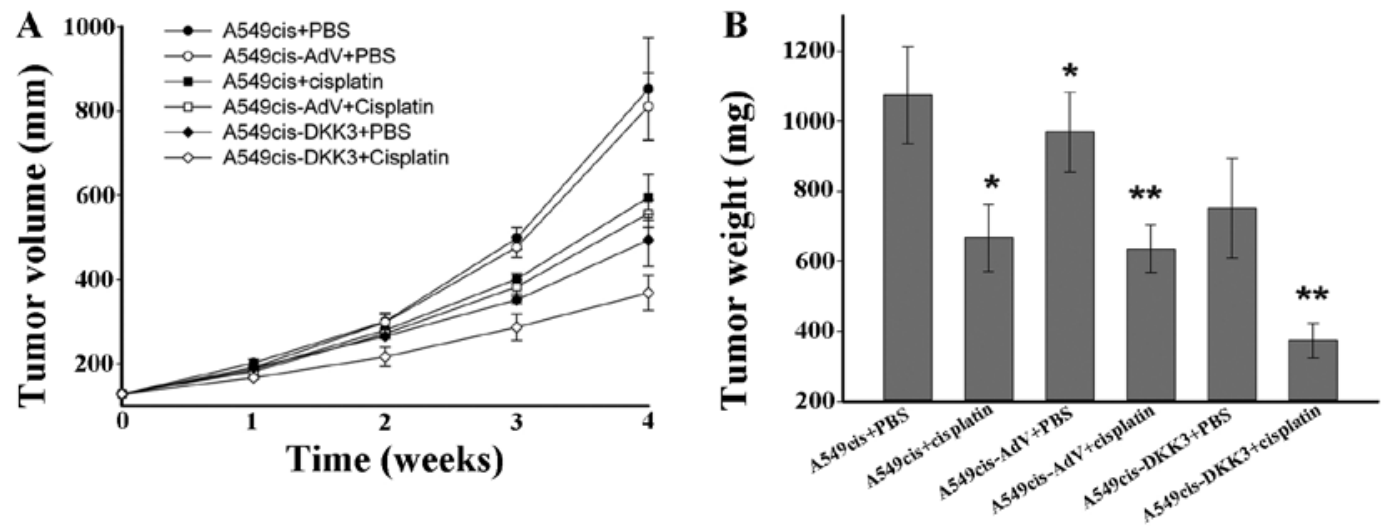

Figure 7. DKK3 transfection inhibits cisplatin-resistant lung cancer cell growth in vivo. (A) Time-tumor volume curve in the xenograft models. (B) Tumor weight at the end of 4 weeks in the xenograft models. Data are representative of 7-9 animals in each conditions. ${ }^{*} \mathrm{P}<0.05 ;{ }^{* *} \mathrm{P}<0.01$. DKK3, Dickkopf-3.

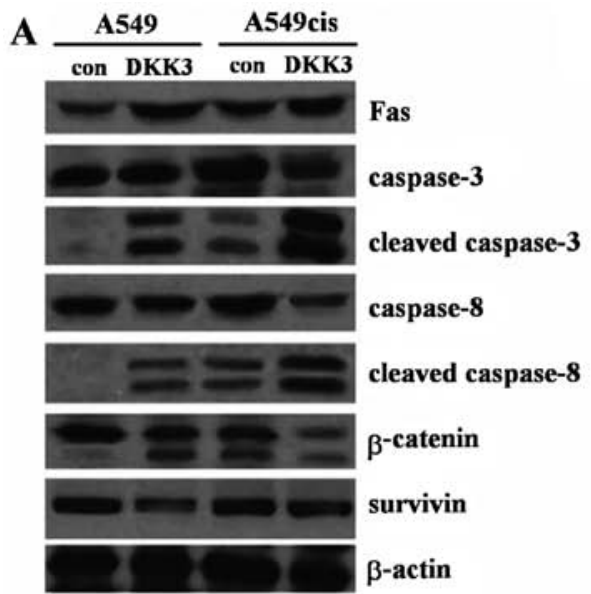

B

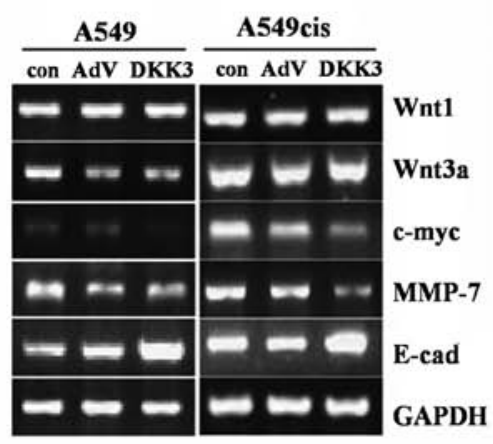

C

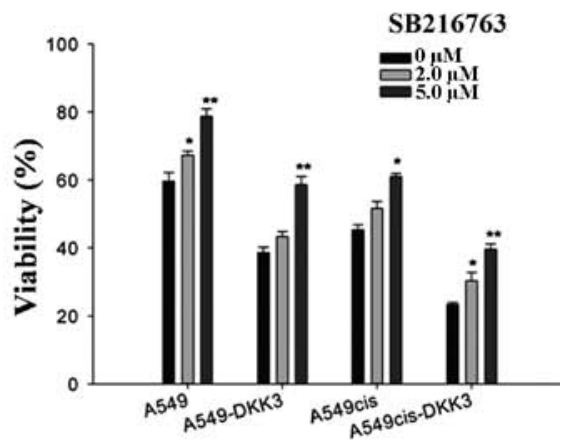

Figure 8. DKK3 transfection regulated survivin and other genetic expressions in A549 lung cancer cells. (A) Western blot analysis. DKK3 transfection resulted in downregulation of the survivin, cyclin D1 and $\beta$-catenin proteins, but an increase in E-cadherin and phosphorylated $\beta$-catenin. (B) RT-PCR analysis. DKK3 transfection was responsible for a marked decrease of MMP-7 and c-myc, and an increase of E-cadherin and $\beta$-catenin, although fewer changes were evident for WNT1 and WNT3a expression. (C) Viability curve of A549cis and A549cis-DKK3 cells pretreated with SB216763 for $2 \mathrm{~h}$ and then treated with cisplatin $(1 \mu \mathrm{M})$ for $72 \mathrm{~h}$. Data are representative of three independent experiments. ${ }^{*} \mathrm{P}<0.05$. DKK3, Dickkopf-3.

mostly at the cytoplasm, and elevated in A549cis lung cancer cells, as shown at immunofluorescence (green) (Fig. 6A). DKK3 transgene increased the sensitivity to cisplatin in A549cis cells (Fig. 6B and C). The dose of cisplatin employed in the xenograft tumor model was optimized by a dose-finding pre-study, in which xenografted tumor-bearing mice were treated with cisplatin $(0.5,1.0,2.0,4.0$ or $8.0 \mathrm{mg} / \mathrm{kg} / \mathrm{day})$ by i.p. injection of $1 \times 10^{6}$ A549cis cells. The mice treated with $2.0 \mathrm{mg} / \mathrm{kg} / \mathrm{day}$ of cisplatin showed a moderate tumor growth delay and retained a survival rate of $\sim 60 \%$ to the 4 -week treatment. The dose of cisplatin was then optimized to $2.0 \mathrm{mg} / \mathrm{kg} / \mathrm{day}$, once a day, 5 days a week for an additional consecutive 4 weeks. Nude mice were randomized to six groups: the A549cis+PBS, the A549cis+cisplatin, the A549-AdV+PBS, the A549cis-AdV+cisplatin, the A549cis-DKK3+PBS and the A549cis-DKK3+cisplatin groups. The tumor growth in vivo was monitored daily and tumor volume and weight were measured. As shown in Fig. 7, tumor growth was more significantly retarded in the A549cis-DKK3+cisplatin group than the A549cis-AdV+cisplatin and A549cis-DKK3+PBS groups, suggesting a synergistic antitumor effect of DKK3 transfection with cisplatin in cisplatin-resistant lung cancer cells in vivo.

DKK3 inhibits survivin expression via the Wnt/ $\beta$-catenin pathway in lung cancer cells. DKK3 transfection significantly enhanced the expression of DKK3. DKK3 was localized at the cytoplasm. To analyze the impact of DKK3 transfection on Wnt signaling, we compared the genes and/or proteins controlled by Wnt signaling or associated with cell cycle, growth and invasion in cultured A549 cells, as shown in Fig. 8A and B. DKK3 suppressed Wnt signaling through $\beta$-catenin degradation, but not directly through Wnt. As shown by the western blot analysis, DKK3 inhibited $\beta$-catenin expression and simultaneously enhanced phosphorylation of $\beta$-catenin (Fig. 8A). DKK3 transfection also downregulated the expression of survivin (Fig. 8A). Results of the RT-PCR assay, showed that DKK3 overexpression was responsible for a decrease of c-myc and MMP7, a moderate increase of E-cadherin and a mild increase of $\beta$-catenin, although fewer changes for the WNT1 and WNT3a expression were evident (Fig. 8B). To analyze the effect of GSK3 $\beta$ inactivation on the concentration-effect 
curves with cisplatin, A549cis and A549cis-DKK3 cells were treated with a GSK3 $\beta$ inhibitor SB216763 $2 \mathrm{~h}$ prior to cisplatin exposure. Viability was assayed $72 \mathrm{~h}$ later. SB216763 treatment is dose-dependently associated with an increased viability in the two cell types (Fig. 8C).

\section{Discussion}

Cisplatin chemotherapy is sensitized by antagonizing aberrantly activated Wnt signaling in lung cancer. Incubation with anti-DKK1 antibody induces apoptosis in A549 cells through the caspase-dependent pathway in vitro and suppresses the growth of A549 and H2170 lung cancer cells in nude mice (23). The tumor suppressor role of Dkk3 in NSCLC is twofold: DKK3 knockdown accelerates NSCLC cell proliferation, whereas Dkk3 overexpression hinders the growth of NSCLC cells through the induction of apoptosis and cell cycle disturbance (15-18). It is thus conceivable that Dkk3, a Wnt inhibitor harboring tumor-suppressive activity, may exert similar functions such as its homologue Dkk1, which hinders NSCLC cell growth synergistically with cisplatin (18). To the best of our knowledge however, the present study is the first one to show an association of Dkk3 and cisplatin resistance in lung cancer. This association is demonstrated by: i) cisplatin-resistant NSCLC cells expressing low levels of DKK3 (Fig. 1); ii) siRNA-mediated knockdown of DKK3 decreasing the sensitivity of NSCLC cells to cisplatin (Fig. 2); iii) DKK3 transfection enhancing cisplatin treatment by inhibiting the growth of and inducing apoptosis in cisplatin-resistant NSCLC cells (Figs. 6 and 7).

Dkk3 is also known as the 'reduced expression in immortalized cells' (REIC). The dysregulated functioning status of Dkk3 contributes to carcinogenesis in the lung, as DKK3 is downregulated in immortalized NSCLC cell lines and human lung cancer tissues (11). DKK3 hypermethylation is universal among lung cancer cell lines and human lung tissue samples and is associated with a poor prognosis $(15,16,24)$. Our data show that Dkk3 expression is further suppressed in NSCLC cell lines with chemoresistance obtained by chronic cisplatin exposure, however the manner in which Dkk3 is reduced is unclear. One possible explanation is the feedback modulating the $\mathrm{Wnt} / \beta$-catenin pathway, i.e., the $\mathrm{Wnt} / \beta$-catenin pathway is activated in cisplatin resistance and, activation of $\mathrm{Wnt} / \beta$-catenin signaling in turn reduces DKK1 expression (25). It is unknown whether DKK3 expression is also negatively regulated by $\mathrm{Wnt} / \beta$-catenin signaling, however the determinant mechanism should be investigated (10).

The role of Wnt/Dkk3 signaling has been well documented in chemoresistance. To the best of our knowledge, for the first time it has been shown that Dkk3 enhances apoptosis and retards growth alone or synergistically with cisplatin in resistant NSCLC cells. This is explained by the direct induction of caspases and indirect inhibition of apoptosis antagonists, with both mechanisms being Wnt-dependent. This study has shown that Wnt inhibition by Dkk3 increased caspase- $3,-8$ and -9 and decreased c-myc, cyclin D1 and survivin in NSCLC cells. Wnt1 inhibited cytochrome $c$ release and subsequent caspase-9 activation in colorectal cancer cells treated with chemotherapeutic agents, rendering them resistant to chemotherapy-mediated apoptosis, whereas chemosensitivity was regained through transcriptional blockage using a dominant-negative mutant of Tcf-4 (26). c-Myc is a nuclear phosphoprotein that responds directly to mitogenic signals and plays a critical role in the cell-cycle progression, particularly during the transition from the G1 to the S phase (27). Wnt signaling promotes oncogenic transformation by inhibiting c-myc-regulated apoptosis $(28,29)$. Cyclin D1 is a rate-limiting signal in the G1-S phase transition which controls the length of the cellular proliferation cycle. Wnt activation triggers the excessive expression of cyclin D1, which has been observed in lung cancer cells separated from patients as well as in immortalized cell lines, and is responsible for rapid growth and proliferation in lung cancer cells $(30,31)$. Survivin, also known as the baculoviral inhibitor of apoptosis repeat-containing 5 (BIRC5), is a member of the inhibitor of apoptosis (IAP) family identified in the majority of human tumors. Survivin binds with caspase- 3 and -7 and attenuates Bax-, Fas- and etoposide-induced apoptosis in cancer cell lines $(32,33)$. As it is abnormally transactivated in lung cancer cells, survivin has been used in the clinic as a prognostic factor and a therapeutic target using antisense oligonucleotides, siRNA, ribozymes, immunotherapy and small molecules (34). Antisense oligonucleotides targeting survivin induces apoptosis and sensitize lung cancer cells to chemotherapy (35). $\mathrm{Wnt} / \beta$-catenin signaling promotes survivin expression, as a Wnt2 antibody induces apoptosis in A549 cells via the inactivation of survivin (36). Cisplatin is a cytotoxic agent targeting DNA, which suppresses RNA transcription and induces cell-cycle derangement and apoptosis. Cisplatin resistance in vivo is largely attributed to increased DNA repair, imbalance of pro-apoptotic-anti-apoptotic signals, which are mediated by Wnt signaling as aforementioned $(37,38)$. The current study shows that Dkk3 downregulated survivin in lung cancer cells via Wnt inhibition, which was accompanied with an elevated expression of pro-apoptotic caspase-3, -8 and -9 and may account for the apoptosis-inducing capabilities of Dkk3. This, Wnt blockage by Dkk3 results in a shift favoring a pro-apoptotic protein expression and discouraging anti-apoptotic proteins, particularly survivin, which disrupts the pro-apoptotic-anti-apoptotic balance and accounts for the chemosensitizing activity of Dkk3.

It is noteworthy that Wnt signaling defines the fate of cancer stem cells (39). Wnt/ $\beta$-catenin signaling controls proliferation, clone formation, migration and drug resistance abilities as well as the expression of cyclin D1 and OCT-4 in A549-derived lung cancer stem cells (40). In addition, survivin expression is downregulated by $\beta$-catenin/TCF- 4 signaling inhibition with APC, another Wnt inhibitor, which increases apoptosis of cancer stem cells and other proliferative cells in the lower crypt, thereby preventing the expansion of cancer stem cells and initiation of colon carcinogenesis (41). Cancer stem cells are considered to be refractory to chemotherapy and radiotherapy, which largely results in the treatment failure of cancer (42). In this regard, the inhibition of Wnt $/ \beta$-catenin signaling by Dkk3 may change the style of survivin expression as well as proliferation, survival and chemosensitivity of lung cancer stem cells. This is an important issue requiring further investigations, as our experiment does not provide direct rationale thereof. 
The Dkk family members include Dkk1,2, 3 and 4, which may be divided into two groups. While Dkk2 activates the Wnt/ $\beta$-catenin signaling pathway (the canonical Wnt signaling pathway), Dkk1, 3 and 4 inactivate Wnt signaling. Dkk3 primarily binds with LRP5/6 and acts as an antagonist of the Wnt/ $\beta$-catenin signaling pathway (the canonical Wnt signaling pathway) $(4,10,18)$. It is also reported that Dkk3 inhibits other Wnt transduction pathways such as the Wnt/JNK signaling pathway in tumor cells (43). In the present study, DKK3 overexpression attenuated $\beta$-catenin nuclear translocation, as well as the expression of c-myc, cyclin D1 and survivin, which are downstream genes controlled by $\mathrm{Wnt} / \beta$-catenin/TCF (LEF) signaling. In addition, the chemosensitizing effect of DKK3 was blocked by Wnt activation with a GSK3 inhibitor SB216763 which stabilizes $\beta$-catenin prior to cisplatin exposure (Fig. 8C). The overall data demonstrate that Dkk3 sensitized cisplatin fundamentally through inhibition of the Wnt/ $\beta$-catenin signaling pathway. However, this does not sufficiently exclude other mechanisms, since a number of apoptotic signaling pathways are involved in the progression of lung cancer $(37,38)$. In another respect, Dkk3 regulates cell proliferation and apoptosis in a complex manner, as Dkk3 may also directly or indirectly modulate apoptosis via the mitochondrial pathway and c-Jun N-terminal kinase (JNK) signaling pathway (44-46). The genome-wide assay has also shown that Wnt inhibition by $\beta$-catenin knockout alters over 130 gene expressions and regulates the PI3K/Akt, NF- $\kappa \mathrm{B}$ and p53 pathways which play a crucial role in cell apoptosis (47). Additional studies may identify the exact mechanism of Dkk3 involved in the reversal of cisplatin resistance.

Results in this study are controversial. Although considered a tumor suppressor, the inactivation of Dkk3 alone is not sufficient for tumor initiation as the knockout of Dkk3 in mice did not increase tumor incidence (48). Moreover, instead of favoring tumor growth as assumed, Dkk3 knockdown favors apoptosis as in the case of Dkk3 overexpression in NSCLC cells $(10,15-18,49)$. According to Jung et al, siRNA-mediated DKK3 knockdown enables H460 cells to detach from the bottom of the culture plate, as well as to initiate the apoptotic process as marked by an increase of cyclin-dependent kinases (CDK) D1 and E. DKK3 knockdown also increases the intracellular levels of reactive oxygen species (ROS) and the expression of P53, p21 and Bax (49). These results suggest that DKK3 with undetectable levels may act as a pro-apoptotic signal by regulating the ROS-mediated expression of P53 and other pro- or anti-apoptotic proteins. The inconsistency between different studies necessitates future investigations to delineate the mechanisms by which Dkk3 regulates Wnt/ $\beta$-catenin signaling as well as chemosensitivity. Efforts should be made to narrow the gap between building a therapeutic target in the bench (animal and cell) study, and the eventual usage in the bedside towards NSCLC.

Taken together, we report on the effect of Dkk3 synergistically with cisplatin on inhibiting NSCLC cell growth, motility in soft agar and inducing apoptosis via inhibition of the Wnt/ $\beta$-catenin signaling pathway. This effect is possibly attributed to the reactivation of apoptotic pathways by the attenuation of survivin expression. Based on its chemosensitizing potential, Dkk3 is a promising target for the development of therapeutic and preventive strategies against cisplatin-resistant
NSCLC. Studies should also be conducted to delineate the exact action mechanisms and to realize the beneficial use of Dkk3 clinically in patients with cisplatin-resistant NSCLC.

\section{Acknowledgements}

The present study was sponsored by the Chinese Ministry of Education Grant WKJ-2009-2-013, and a research fund from the Ministry of Health of Henan Province. The authors would like to thank Zhao-Gang Dong and Li Zhang for their technical support and kind review, and of the assistance on the manuscript.

\section{References}

1. Jemal A, Siegel R, Xu J and Ward E: Cancer statistics, 2010. CA Cancer J Clin 60: 277-300, 2010.

2. Spira A and Ettinger DS: Multidisciplinary management of lung cancer. N Engl J Med 350: 379-392, 2004.

3. de las Peñas R, Sanchez-Ronco M, Alberola V, et al: Polymorphisms in DNA repair genes modulate survival in cisplatin/ gemcitabine-treated non-small-cell lung cancer patients. Ann Oncol 17: 668-675, 2006.

4. Logan CY and Nusse R: The Wnt signaling pathway in development and disease. Annu Rev Cell Dev Biol 20: 781-810, 2004.

5. Mazieres J, He B, You L, Xu Z and Jablons DM: Wnt signaling in lung cancer. Cancer Lett 222: 1-10, 2005.

6. Klaus A and Birchmeier W: Wnt signalling and its impact on development and cancer. Nat Rev Cancer 8: 387-398, 2008.

7. Wang H, Fan L, Xia X, et al: Silencing Wnt2B by siRNA interference inhibits metastasis and enhances chemotherapy sensitivity in ovarian cancer. Int J Gynecol Cancer 22: 755-761, 2012.

8. Dowejko A, Bauer R, Bauer K, Müller-Richter UD and Reichert TE: The human HECA interacts with cyclins and CDKs to antagonize Wnt-mediated proliferation and chemoresistance of head and neck cancer cells. Exp Cell Res 318: 489-499, 2012.

9. Su HY, Lai HC, Lin YW, et al: Epigenetic silencing of SFRP5 is related to malignant phenotype and chemoresistance of ovarian cancer through Wnt signaling pathway. Int J Cancer 127: 555-567, 2010.

10. $\mathrm{Li} \mathrm{Y}$ and $\mathrm{Bu} \mathrm{G}$ : LRP5/6 in Wnt signaling and tumorigenesis. Future Oncol 1: 673-681, 2005.

11. Tsuji T, Miyazaki M, Sakaguchi M, Inoue $\mathrm{Y}$ and Namba M: A REIC gene shows down-regulation in human immortalized cells and human tumor-derived cell lines. Biochem Biophys Res Commun 268: 20-24, 2000.

12. Ueno K, Hirata H, Majid S, et al: Wnt antagonist DICKKOPF-3 (Dkk-3) induces apoptosis in human renal cell carcinoma. Mol Carcinog 50: 449-457, 2011.

13. Mizobuchi Y, Matsuzaki K, Kuwayama K, et al: REIC/Dkk-3 induces cell death in human malignant glioma. Neuro Oncol 10: 244-253, 2008.

14. Hoang BH, Kubo T, Healey JH, et al: Dickkopf 3 inhibits invasion and motility of Saos-2 osteosarcoma cells by modulating the Wnt- $\beta$-catenin pathway. Cancer Res 64: 2734-2739, 2004.

15. Hsieh SY, Hsieh PS, Chiu CT and Chen WY: Dickkopf-3/REIC functions as a suppressor gene of tumor growth. Oncogene 23: 9183-9189, 2004

16. Yue W, Sun Q, Dacic S, et al: Downregulation of Dkk3 activates $\beta$-catenin/TCF-4 signaling in lung cancer. Carcinogenesis 29: 84-92, 2008

17. Tsuji T, Nozaki I, Miyazaki M, et al: Antiproliferative activity of REIC/Dkk-3 and its significant down-regulation in non-small-cell lung carcinomas. Biochem Biophys Res Commun 289: 257-263, 2001.

18. Veeck $\mathbf{J}$ and Dahl E: Targeting the Wnt pathway in cancer: the emerging role of Dickkopf-3. Biochim Biophys Acta 1825: 18-28, 2012.

19. Gosepath EM, Eckstein N, Hamacher A, et al: Acquired cisplatin resistance in the head-neck cancer cell line Cal27 is associated with decreased DKK1 expression and can partially be reversed by overexpression of DKK1. Int J Cancer 123: 2013-2019, 2008. 
20. Shou J, Ali-Osman F, Multani AS, Pathak S, Fedi P and Srivenugopal KS: Human Dkk-1, a gene encoding a Wnt antagonist, responds to DNA damage and its overexpression sensitizes brain tumor cells to apoptosis following alkylation damage of DNA. Oncogene 21: 878-889, 2002.

21. Hong WS, Saijo N, Sasaki Y, et al: Establishment and characterization of cisplatin-resistant sublines of human lung cancer cell lines. Int J Cancer 41: 462-467, 1988.

22. Coghlan MP, Culbert AA, Cross DA, et al: Selective small molecule inhibitors of glycogen synthase kinase-3 modulate glycogen metabolism and gene transcription. Chem Biol 7: 793-803, 2000.

23. Sato N, Yamabuki T, Takano A, et al: Wnt inhibitor Dickkopf-1 as a target for passive cancer immunotherapy. Cancer Res 70: 5326-5336, 2010

24. Suzuki M, Shigematsu H, Nakajima T, et al: Synchronous alterations of Wnt and epidermal growth factor receptor signaling pathways through aberrant methylation and mutation in non-small cell lung cancer. Clin Cancer Res 13: 6087-6092, 2007.

25. Niida A, Hiroko T, Kasai M, et al: DKK1, a negative regulator of Wnt signaling, is a target of the $\beta$-catenin/TCF pathway. Oncogene 23: 8520-8526, 2004.

26. Chen S, Guttridge DC, You Z, et al: Wnt-1 signaling inhibits apoptosis by activating $\beta$-catenin/T cell factor-mediated transcription. J Cell Biol 152: 87-96, 2001.

27. Spencer CA and Groudine M: Control of c-myc regulation in normal and neoplastic cells. Adv Cancer Res 56: 1-48, 1991.

28. You Z, Saims D, Chen S, et al: Wnt signaling promotes oncogenic transformation by inhibiting c-Myc-induced apoptosis. J Cell Biol 157: 429-440, 2002.

29. He TC, Sparks AB, Rago C, et al: Identification of c-MYC as a target of the APC pathway. Science 281: 1509-1512, 1998.

30. Tetsu $\mathrm{O}$ and McCormick F: $\beta$-Catenin regulates expression of cyclin D1 in colon carcinoma cells. Nature 398: 422-426, 1999.

31. Hirata $\mathrm{H}$, Hinoda $\mathrm{Y}$, Nakajima $\mathrm{K}$, et al: Wnt antagonist $D K K 1$ acts as a tumor suppressor gene that induces apoptosis and inhibits proliferation in human renal cell carcinoma. Int J Cancer 128: 1793-1803, 2011.

32. Perkins C, Kim CN, Fang G and Bhalla KN: Arsenic induces apoptosis of multidrug-resistant human myeloid leukemia cells that express $\mathrm{Bcr}-\mathrm{Abl}$ or overexpress MDR, MRP, $\mathrm{Bcl}-2$, or $\mathrm{Bcl}-\mathrm{x}_{\mathrm{L}}$ Blood 95: 1014-1022, 2000.

33. Ambrosini G, Adida C, Sirugo G and Altieri DC: Induction of apoptosis and inhibition of cell proliferation by survivin gene targeting. J Biol Chem 273: 11177-11182, 1998.

34. Ryan BM, O'Donovan N and Duffy MJ: Survivin, a new target for anti-cancer therapy. Cancer Treat Rev 35: 553-562, 2009.
35. Olie RA, Simões-Wüst AP, Baumann B, et al: A novel antisense oligonucleotide targeting survivin expression induces apoptosis and sensitizes lung cancer cells to chemotherapy. Cancer Res 60: 2805-2809, 2000.

36. You L, He B, Xu Z, et al: Inhibition of Wnt-2-mediated signaling induces programmed cell death in non-small-cell lung cancer cells. Oncogene 23: 6170-6174, 2004.

37. Siddik ZH: Cisplatin: mode of cytotoxic action and molecular basis of resistance. Oncogene 22: 7265-7279, 2003.

38. Singhal S, Miller D, Ramalingam S and Sun SY: Gene expression profiling of non-small cell lung cancer. Lung Cancer 60: 313-324, 2008.

39. He B and Jablons DM: Wnt signaling in stem cells and lung cancer. Ernst Schering Found Symp Proc 2006: 27-58, 2006.

40. Teng Y, Wang X, Wang Y and Ma D: Wnt $/ \beta$-catenin signaling regulates cancer stem cells in lung cancer A549 cells. Biochem Biophys Res Comm 392: 373-379, 2010.

41. Zhang T, Otevrel T, Gao Z, et al: Evidence that APC regulates survivin expression: a possible mechanism contributing to the stem cell origin of colon cancer. Cancer Res 61: 8664-8667, 2001.

42. Kim CF, Jackson EL, Woolfenden AE, et al: Identification of bronchioalveolar stem cells in normal lung and lung cancer. Cell 121: 823-835, 2005

43. Niehrs C: Function and biological roles of the Dickkopf family of Wnt modulators. Oncogene 25: 7496-7481, 2006.

44. Yang ZR, Dong WG, Lei XF, Liu M and Liu QS: Overexpression of Dickkopf-3 induces apoptosis through mitochondrial pathway in human colon cancer. World J Gastroenterol 18: 1590-1601, 2012.

45. Abarzua F, Sakaguchi M, Takaishi M, et al: Adenovirus-mediated overexpression of REIC/Dkk-3 selectively induces apoptosis in human prostate cancer cells through activation of c-Jun- $\mathrm{NH}_{2}$-kinase. Cancer Res 65: 9617-9622, 2005.

46. Kashiwakura Y, Ochiai K, Watanabe M, et al: Down-regulation of inhibition of differentiation-1 via activation of activating transcription factor 3 and Smad regulates REIC/Dickkopf-3-induced apoptosis. Cancer Res 68: 8333-8341, 2008.

47. Huang M, Wang Y, Sun D, et al: Identification of genes regulated by Wnt/ $\beta$-catenin pathway and involved in apoptosis via microarray analysis. BMC Cancer 6: 221-221, 2006.

48. Barrantes Idel B, Montero-Pedrazuela A, Guadaño-Ferraz A, et al: Generation and characterization of dickkopf3 mutant mice. Mol Cell Biol 26: 2317-2326, 2006

49. Jung IL, Kang HJ, Kim KC and Kim IG: Knockdown of the Dickkopf 3 gene induces apoptosis in a lung adenocarcinoma. Int J Mol Med 26: 33-38, 2010. 